Test-Interaction (ANTI) by Callejas et al (2004). Generally, these tasks measure the functioning of the Alerting network by means of a phasic alertness task, that is, measuring the reduction in reaction time provided by a warning signal in comparison to when this signal is not available. However, this measure may provide only a partial estimation of the functioning of the Alertness network, as the vigilance function of attention is not analysed in these tasks. The present work modifies the original ANTI task to add a measure of vigilance, thus attempting to obtain a more complete estimation of the functioning of the Alertness network. Participants now have to detect an infrequent event while performing a variation of the ANTI task so that some indicators of vigilance can be obtained. These data are analysed according to the STD (Signal Detection Theory) and the measures of $d^{\prime}$ and ?for sensitivity and decision processes are obtained throughout the time taken to perform the task. In addition, the usual Alerting, Orienting and Executive Control network measures are obtained, together with the usual interaction. The results are compared with those found in the original task and implications for safety driving are highlighted.

\title{
References
}

Callejas A, Lupianez $\mathrm{J}$ and Tudela P. The three attentional networks: On their independence and interactions. Brain and Cognition 2004;54, 225-7.

Grier RA, Warm JS, Dember GM, Galinsky T, Szalma JL and Parasuraman R. The Vigilance Decrement Reflects Limitations in Effortful Attention, Not Mindlessnesss Human Factors. 2004;45:349-58.

Fan J, McCandliss BD, Sommer T, Raz A and Posner MI. Testing the efficiency and independence of attentional networks. Journal of Cognitive Neuroscience, 2002;14:340-47.

Posner MI. Chronometric explorations of mind. 1978; Hillsdale NJ: Erlbaum.

\section{THE ROLE THAT THE THREE ATTENTIONAL NETWORKS AND THE INFREQUENT TARGETS DETECTION PLAY IN SAFELY DRIVING}

C Castro* Correspondence: Faculty of Psychology, University of Granada, Campus Cartuja, s/n 18071 Granada 18071, Spain

\subsection{6/ip.2010.029215.978}

Different tasks have been developed to obtain an individual measure of the functioning of the three attentional networks (Alerting, Orienting and Executive Control by Posner and Petersen's model), such as the Attentional Networks Test (ANT) by Fan et al (2002) and the Attentional Networks 